\title{
Machine Vision-Based Pilling Assessment: A Review
}

\author{
Rocco Furferi, PhD, Lapo Governi, PhD, Yary Volpe, PhD \\ University of Florence (Italy), Department of Industrial Engineering, Firenze ITALY \\ Correspondence to: \\ Rocco Furferi email: rocco.furferi@unifi.it
}

\begin{abstract}
Pilling is an undesired defect of textile fabrics, consisting of a surface characterized by a number of roughly spherical masses made of entangled fibers. Mainly caused by the abrasion of fabric surface occurring during washing and wearing of fabrics, this defect needs to be accurately controlled and measured by companies working in the textile industry. Pilling measurement is traditionally performed using manual procedures involving visual control of fabric surface by human experts. Since the early nineties, great efforts in developing automatic and non-intrusive methods for pilling measurement have been made all around the world with the final aim of overcoming traditional, visual-based and subjective procedures. Machine Vision proved to be among the best options to perform such defect assessment since it provided increasingly performing measurement equipment and tools, serving the purpose of automatic control. In particular, a relevant number of interesting works have been proposed so far, sharing the idea of helping (or even replacing) traditional measurement methods using image processing-based ones. The present work provides a rational and chronological review of the most relevant methods for pilling measurement proposed so far. This work serves the purposes of 1) understanding whether today's automatic machine vision-based pilling measurement techniques are ready for supplanting traditional pilling measurement and 2) providing textile technology researchers with a bird's eye view of the main methods studied to confront with this problem.
\end{abstract}

Keywords: Review, Fabrics, Pilling assessment, Machine Vision, Image Processing, Artificial Neural Networks.

\section{INTRODUCTION}

As widely recognized [1], the term "pilling" is referred to a surface defect occurring in textile fabrics and consisting of entangled fibers forming the so called "pills". Such pills are, usually, caused by the combination of washing and wearing of fabrics; in detail, due to the abrasion of fabric surface, a number of loose fibers tend to entangle into short fine hair, thus developing into spherical bundles anchored to the surface of the fabric (see Figure 1).

Fabric's pill formation (i.e. the so called "resistance to pilling") is typically measured using procedures described in Standards such as the D4970/D4970M10e1 (ASTM, 2010) and the UNI EN ISO 129452004; since fabrics take a long time to be pilled in normal use, resistance to pilling needs to be tested by a simulated accelerated wear, followed by a visual assessment of the degree of pilling based on a visual comparison of the sample to a set of test images.

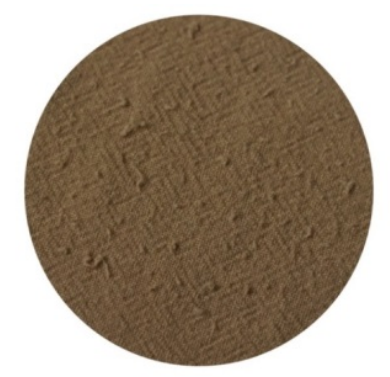

FIGURE 1. Example of pilled fabric.

Two common pieces of equipment for pilling measurement, mainly used in Europe, are the Martindale pilling tester and the Pilling Box.

The Martindale tester consists of a number of testing plates (See Figure 2) on which the abrading fabrics is attached; these four testing plates are mounted on the base plate of the instrument. Generally speaking, fabrics to be tested using Martindale are cut in an approximate circular shape with diameter equal to $90 \pm 1 \mathrm{~mm}$. A worsted wool cloth is used for abrading the samples and a trajectory based on the Lissajous figure is used to perform each cycle (more precisely, a cycle consists of 16 movements in the Lissajous figure). A $12 \mathrm{kPa}$ head pressure is applied by the machine. 


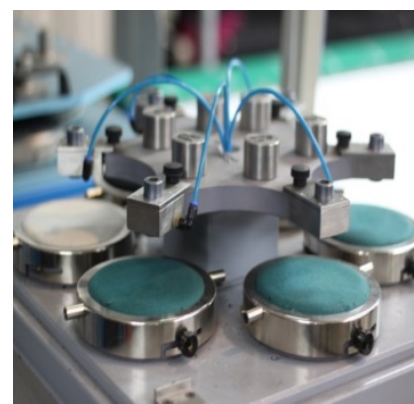

FIGURE 2. Martindale pilling tester.

In Pilling Box (see Figure 3) samples are mounted on polyurethane tubes and are tumbled in cork-lined rotating wooden boxes. Accordingly, the samples move under the condition of no pressure and the specimens are conducted under mutual transient touching. As a consequence, unlike the Martindale method, the rubbing for the samples is random.

Whichever the device (Martindale or Pilling Box), the final result consists of abraded fabrics to be assessed in terms of pilling. This is performed by skilled operators (experts) comparing the specimens, after a predefined number of cycles performed by the testing equipment, with visual standards (which may be actual fabrics samples or photographs). On the basis this comparison, the experts define the resistance to pilling using the so called "degree of pilling” [1] i.e. an index varying on a scale ranging from 5 -which means no pilling- to 1 -which means very severe pilling (see Table I).

TABLE I. Degree of pilling.

\begin{tabular}{|c|c|c|}
\hline $\begin{array}{l}\text { Pilling } \\
\text { degree }\end{array}$ & Description & Details according to ISO-12945-2:2000 [66] \\
\hline 1 & $\begin{array}{l}\text { Very severe } \\
\text { pilling }\end{array}$ & Severe pilling covering whole of the fabric surface \\
\hline 2 & Severe pilling & $\begin{array}{l}\text { Distinct surface piling. Pills of various size and } \\
\text { density covering a large proportion of the surface. }\end{array}$ \\
\hline 3 & $\begin{array}{l}\text { Moderate } \\
\text { Pilling }\end{array}$ & $\begin{array}{l}\text { Moderate surface pilling. Pills of varying size and } \\
\text { density partially covering the surface. }\end{array}$ \\
\hline 4 & Low pilling & Slight surface fuzzing \\
\hline 5 & No Pilling & No change in fabric aspect. \\
\hline
\end{tabular}

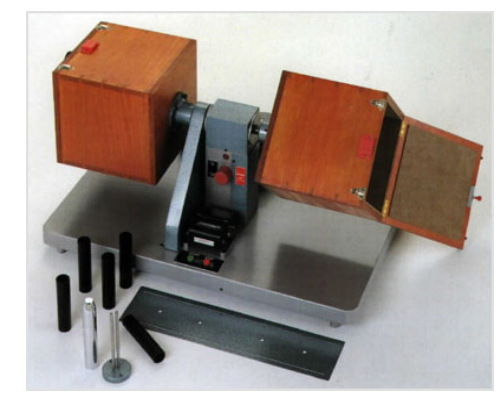

FIGURE 3. Pilling Box.
This method proves to be suitable for predicting the actual behavior of fabrics in everyday use only in some specific conditions. For instance, according to the ASME Standard, laboratory test is considered reliable as an indication of relative end-use performance in cases where the difference in abrasion resistance of various materials is large, but they should not be relied upon in prediction of actual wear-life in specific end uses, unless there are data showing the specific relationship between laboratory abrasion tests and actual wear in the intended enduse.

According to [2], the main drawback of the subjective methods based on estimation by experts is their inconsistency and the inaccuracy of the rating results. Henceforward, there is still today a need for devising objective evaluation methods, relying in automatic and non-intrusive pilling measurement.

With the aim of speeding up the pilling measurement procedure and, at the same time, to increase the reliability of the visual control, in recent years a number of Machine Vision (MV) systems have been proposed in order to overcome the limitations of traditional, visually-based, pilling measurement.

On the basis of the most relevant results obtained in this field, the present paper provides a rational and chronological review of the most promising methods proposed so far. It is the authors' opinion that such a review can help researchers in understanding the working principles of today's best automatic machine vision-based pilling measurement techniques. Moreover, on the basis of the best practices offered by the reviewed works, future trends in pilling measurement are postulated, so that interested researchers are aware of the future scenario that lies ahead for the future.

\section{A Categorization of Methods for Automatic} Fabric Pilling Assessment Using Machine Vision In the last decades automated visual inspection $(A V I)$ of fabrics for quality control faced an increasing trend in the textile industry due to the considerable development of technologies related to vision systems. Several approaches have been proposed in the scientific literature [3-6] employing image processing-based methods and statistical parameters (such as mean, variance and median) for defect detection on fabrics. Pilling measurement using machine vision systems makes no exception: a number of methodologies have been proposed in order to explore automatic or semi-automatic pill detection and classification. Basically (in almost all the methods) the starting point consists of digital 
images of pilled fabrics. These images (representing either pilled fabric specimens to be evaluated or standard reference) are, then, processed in several different ways in order to extract some features describing fabric pilling. Finally, such parameters are used for grading the fabrics or for characterizing their quality. While the starting point and the final results are ultimately shared by all the techniques, what changes is the method adopted for extracting the information used for pilling grading. On the basis of main literature works, in the present work the following categories are identified:

1) $2 \mathrm{D}$ imaging methods based on thresholding.

2) 2D imaging methods based on Fourier and/or Wavelet analysis.

3) 3D imaging methods.

4) AI-based methods (using either $2 \mathrm{D}$ or $3 \mathrm{D}$ images). Understandably, different categories could be used for describing existing works. Moreover some more recent techniques use approaches comprised in more than one of the above categories. Nevertheless, it is the authors' opinion that the given categorization, although open to improvement, is effective for understanding and systematizing the knowledge about how the pilling assessment problem has been faced by more than a few authors all over the world. As already mentioned, possible approaches are presented in a chronological order so that the main improvements brought by researchers are timestreamed.

\section{D Imaging Methods Based on Thresholding}

The main idea of almost all the papers dealing with 2D imaging methods is to perform pill detection using image segmentation [1], the process of partitioning the original image into multiple segments including fabric background and pills. This process is usually, in its turn, aimed at determining parameters such as the number and the density of pills and/or the area occupied by the pills on the fabric surface. Once this task is performed, pilling grade is obtained as a parameter inferred from the number of pills, or by comparing the pilled fabric with a reference fabric (either with or without pills). As a matter of fact, almost all methods classified in this category use, at some point, an image binarization by applying one or more thresholds and, possibly, morphological operations on images. In Figure 4 an exemplificative flow diagram characterizing this category of methodologies is shown.

An early work dealing with image segmentation was carried out by Konda et al. in 1990 [7]; images of fabric samples, pilled using Martindale equipment, were acquired using a commercial camera under near-tangential illumination thus obtaining images with high pill-to-background contrast. Obtained images are then binarized using two different thresholds with the final result of detaching the background from the pills. In Konda's work, the background is represented with black (pixel value equal to 0) while pills are depicted as white blobs (pixel value equal to 1). Eventually, pilling class of the fabric sample under investigation is evaluated from the total number (or total area) of pills.

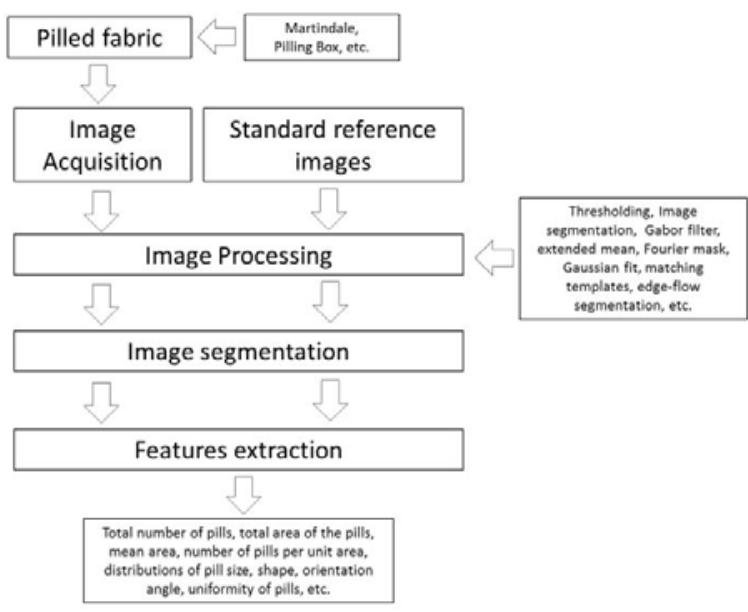

FIGURE 4. Flow diagram of 2D imaging methods based on thresholding.

In Figure 5 an illustrative image from Konda's work describing the number of pills as a function of pill size is proposed.

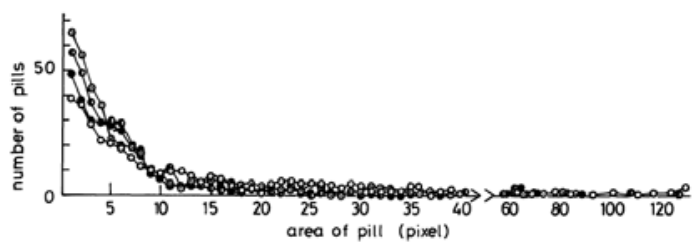

FIGURE 5. Number of pills as a function of pill size: an example from Konda's work [7].

In 1996, Abril et al. [8] used some techniques typical of digital image processing with the aim of evaluating the pilling degree. From the analysis of a set of standard images a sequential method for an objective measurement was devised. An intermediate result of the proposed approach consists of binary image obtained using segmentation by local binarization. In Figure 6 a $64 \times 64$ pixels portion of such binarized image, taken from Abril's work, is shown. 


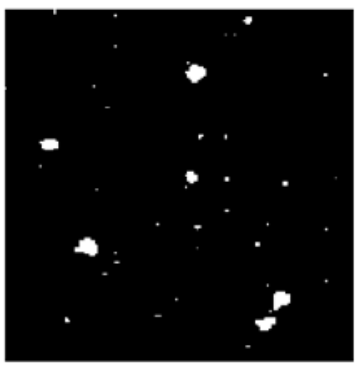

FIGURE 6. Part of a processed image after segmentation by local binarization (Abril et al.[8]).

Starting from the binary image, an evaluation of the total pilled area (for each processed image) to be related with the pilling degree is carried out. In particular, the authors claim that a logarithmic relationship between the total pilling area and the degree of pilling subsists observed (see Figure 7). The proposed method has been further implemented by the same authors in [9] by using 1) a Top-hat transform (an operation that extracts small elements and details from given images [10]) for obtaining background uniformity, 2) an image segmentation based on a Gaussian model [11] of the background, and 3) a selective noise elimination in the binary image. The maximum error of misclassification in percent of background pixels in the total amount of pixels classified as pilling (beyond the threshold) was found equal to $0.3 \%$ for an optimally selected threshold value.

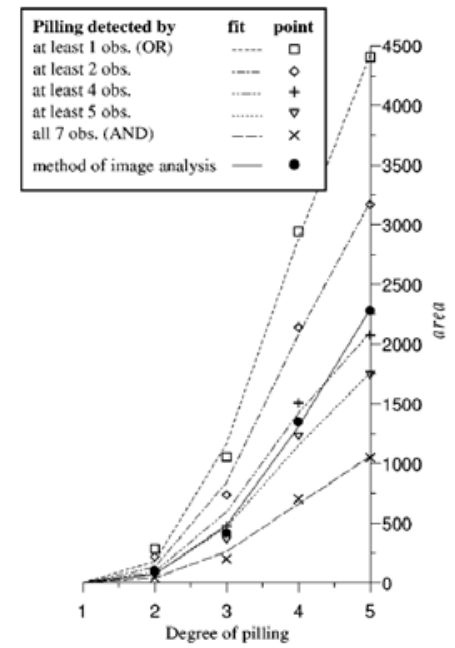

FIGURE 7. Areas of pilling corresponding to the standard images of pilling degree varying in the range [1-5]: comparison between human visual performance and image-processing based method proposed by Abril et al. [8].
In [12] the main concept described in [8] were recalled and a digital image processing was used to determine pills size, number, shape, orientation angle, contrast, total area and the mean area of pills on a fabric surface, especially using thresholding techniques.

A MV-based methodology that automatically counts the number of pills on textile fabric samples and classifies them into pre-defined classes has been proposed in [13]. A CCD camera is used to capture successive gray scale images of the fabric sample; then, segmentation, Radon transform [14], morphological filtering, and de-trending operations [15] are applied to determine the pilling count. Using fuzzy membership functions [16], the fabric pilling count is ultimately related to fabric pilling resistance. A tool developed to detect and describe pills on solidshade fabrics (after being imaged with conventional personal-computer-based hardware) has been devised in 1998 [17]. In such a work, the devised software evaluated the total number, total area, and total volume of fabric pills. Moreover, the system evaluated distributions of pill size, shape, orientation angle, contrast and uniformity of pill spatial distribution on the fabric.

In the same year, Xiaohong and $\mathrm{Mu}$ [18] proposed a method for pilling evaluation where the image of pilled fabric is preprocessed on the basis of image's gray-scale statistical and/or mathematical morphology. The pilling of fabric is, then, assessed synthetically on the basis of the size, the number and the morphology of pilling. Tested with knitted samples, the results proved to be satisfactory.

In 1999, Fazekas et al. [19] located pill regions on fabric samples by combining template matching techniques and image thresholding. Special illumination arrangements, i.e. a bi-directional illumination, were used to grasp the depth information from images, so that pills were properly segmented from the background (see Figure 8).
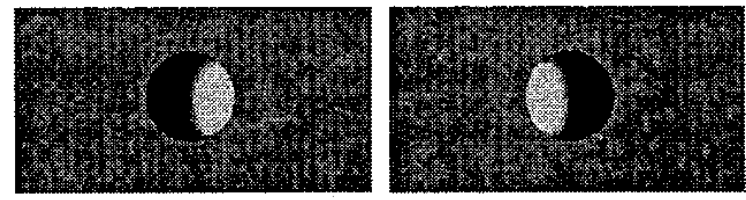

FIGURE 8. Bi-directional illumination used for pill-detection by Fazekas et al [19]. 
Finally, statistically comparing the number of pills detected over a given area with the assessment (quality classification) given by the textile experts, it is possible to empirically determine the optimal threshold values - measured in pills per area between the quality classes defined by the standard.

A remarkable approach to extract pill features from fabric images was proposed in [21 20]; using a twodimensional Gaussian fit theory, authors train a "pill template" using actual pill images and determine a reasonable threshold for image segmentation using a histogram-fitting technique. Using the described approach five parameters to describe pill properties (i.e. pill number, mean area of pills, total area of pills, contrast and density) are defined. Finally, from such data, a definition of pilling grade is provided.

The level of pilling has been also identified and characterized using the size and numbers of the existing pills in 2005 by Huang et al. [21].

Since segmentation algorithms can be affected by fabric texture, color, and pattern, an edge-flow based algorithm taking all these factors into account has been proposed in [22]. This approach can be used in different kinds of fabrics, especially those having complex background. In Figure 9, the pilling segmentation obtained in such a work starting from fine texture woven is shown.

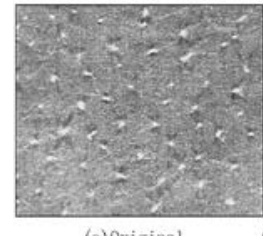

(a) Original

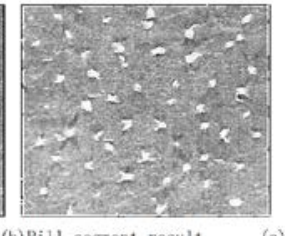

(b) Pill segrent result

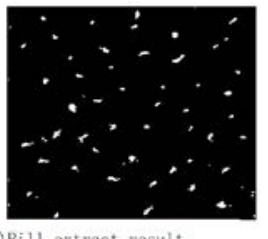

Pill extract result
FIGURE 9. Pilling segmentation performed in [21].

The final result of the proposed method consists of properly segmented images where pills are easy detectable from the background. Awkwardly, no information regarding the pilling grade deriving from image analysis is provided.

A more recent application of image analysis to assess the fabric wrinkle and abrasion resistance in order to compare with experimental methods is described in [23]. By employing an appropriate lighting method, sample images were captured by using a scanner; then, images prepared from samples were processed using MATLAB ${ }^{\circledR}$ in order to extract the pills from the background thus deriving a pilling grade.
In [24] an edge-flow based fabric pilling segmentation algorithm which utilizes image color, texture and phase of the edge flow vector [25] was adopted in order to implement the pilling segmentation of various complex fabrics.

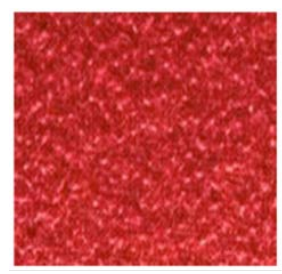

Original image

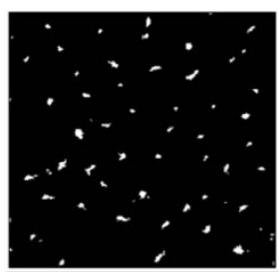

Image after pilling extraction
FIGURE 10. Pilling segmentation performed in [24].

After recognizing the pilling from its background, the total number of pilling can be obtained by searching the connective regions in binary image. As depicted in Figure 11, every connective region is labelled and the total number of non-zero pixel values is calculated.
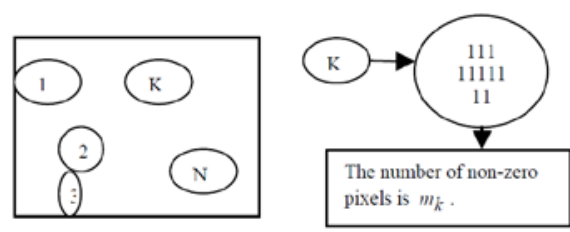

FIGURE 11. A schematic representation of the method for searching pilling regions provided in [24].

The relationships between pilling grades and the total number of pilling, the size of the total area and the optical pilling grading are declared equal to, respectively $0.96,0.94$ and 0.92 .

A novel method for locating the pills in woven fabric based on Gabor filter [26] is proposed in [27]; Gabor filter is applied to pilled fabric images in order to remove fabric textures, thus enhancing the pills. In the enhanced fabric image, threshold method is finally used to segment and locate the fabric pills.

In [28] a method to analyze a pilled knitted fabric surface by using color digital images (RGB model) is proposed. Application of the RGB model for the acquired images (see Figure 12) allows differentiating pilling from fuzzing changes more effectively and precisely with respect to grayscalebased methods. The final result of this approach, whose flow-chart is illustrated in Figure 13, consists of an index $\mathrm{N}$ indicating the percentage of pilled area. 
Such a value is lastly related with the fabric pilling grade. Moreover, the classification of the pilling grade using the $\mathrm{N}$ value for different groups of fabrics is proposed as a future work.

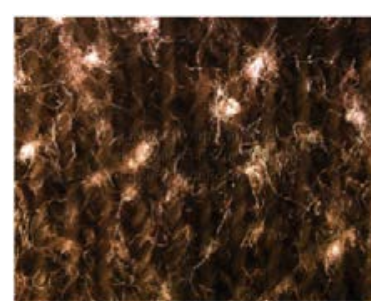

Original RGB image

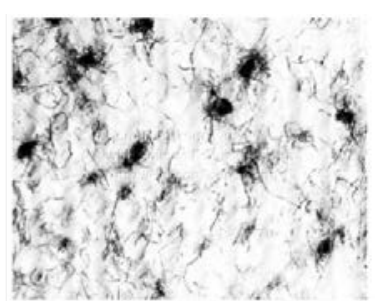

Image after pilling extraction
FIGURE 12. Pilling segmentation performed in [28].

The extended mean shift algorithm was also used to try to solve the segmentation of fabric pilling images in [29] by introducing two main steps: image prefiltering and final segmentation. Laboratory test performed by authors shows that the proposed algorithm can get excellent segmentation if an optimal choice of the three required threshold parameters is assumed.

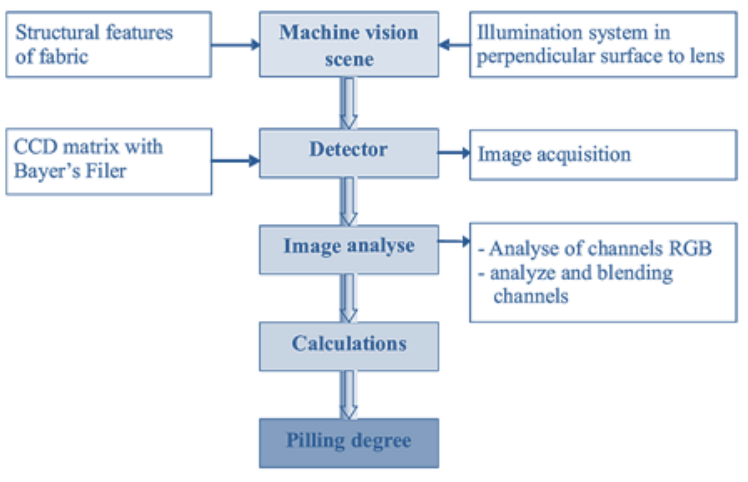

FIGURE 13. Flow-chart of the method proposed in [28].

2D Imaging Methods Based on Fourier Analysis And Wavelet

While the above mentioned papers are mostly based on image thresholding, another range of 2D image processing based methods developed for assessing pilling grade is related to Fourier Transform and Wavelet analysis (see Figure 14).

In 2002, Jensen and Carstensen [30] took an image from fabric surface and used a Fourier mask to filter the knitted stitch background from the fuzz and pill. In particular, the Fourier mask has been used to filter the knitted stitch background from the fuzz and pill.

A pilling measurement cabinet was specifically designed and developed in [31] (see Figure 15).
Captured images were analyzed using appositely developed software based on thresholding and various pilling parameters such as total number of pills, total area of the pills, mean area and number of pills per unit area are measured. Such parameters were, then, compared with the same ones obtained manually, thus showing a good correlation with fabric grading performed by experts.

In particular, the authors demonstrated that the highest is the pilling, the larger is the pills per unit area parameter.

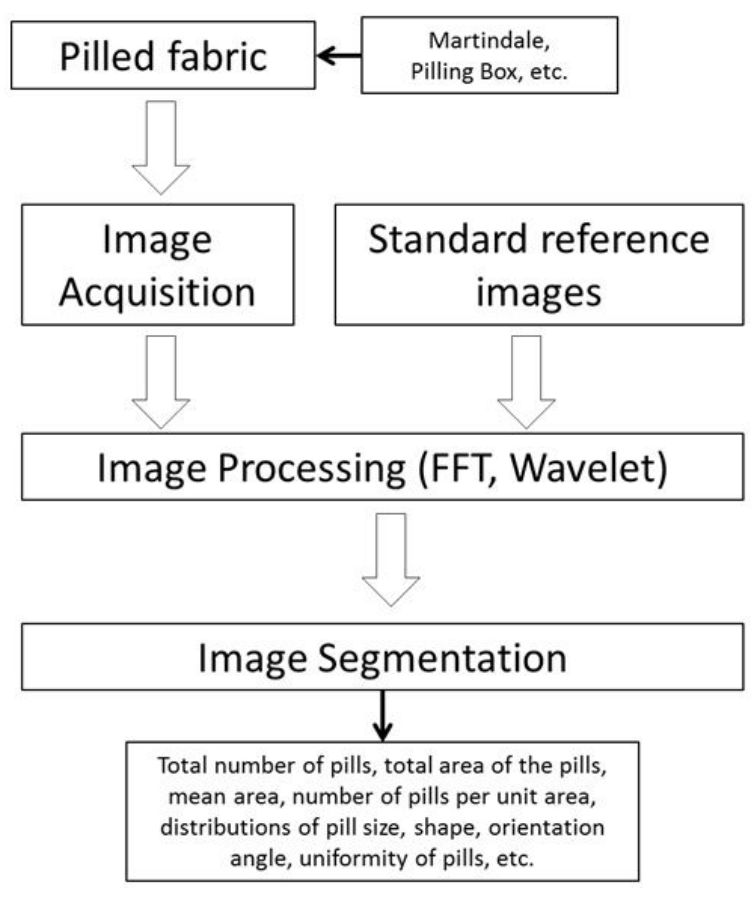

FIGURE 14. Flowchart of 2D imaging methods based on Fourier analysis and Wavelet.

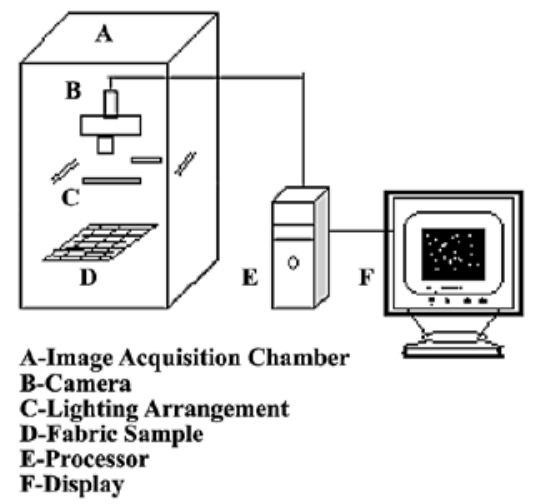

FIGURE 15. Pilling measurement cabinet was specifically designed and developed in [31]. 
In Figure 16 some details about the pilling parameters of standards obtained from the system devised in [31] and the EMPA standards are proposed thus demonstrating the effectiveness of the proposed method.

\begin{tabular}{lcccc}
\hline Grade & Pill count & Area (square inches) & Mean pill area (square inches) & Pills per square inch \\
\hline $1-2$ & 67 & 1.5924 & 0.0238 & 2.8089 \\
23 & 53 & 0.9602 & 0.0181 & 2.2212 \\
$3-4$ & 31 & 0.6640 & 0.0214 & 1.2992 \\
$4-5$ & 5 & 0.0023 & 0.0004 & 0.2095
\end{tabular}

FIGURE 16. Some details about the pilling parameters of standards obtained from the system devised in [31] and the EMPA standards.

A more recent approach to pilling evaluation based on the wavelet reconstruction scheme was investigated in [32]. The method, preliminary evaluated using SM50 European standard pilling images, shows that reconstructed resolution level, wavelet bases and sub-image used for reconstruction affect the segmentation of pills and, thus, pilling grading. The area ratio of pills to total image was successfully used as a pilling rating factor (in analogy with a good number of works belonging to the all the 3 categories mentioned before).

In [33] frequency-domain image processing is used to separate periodic structures in the image (the fabric weave/knit pattern) from non-periodic structures in the image (the pills).

The authors propose that for two-dimensional discrete wavelet transform (2DDWT) analysis of unpilled fabric images, where the wavelet scale is close to the fabric inter-yarn pitch, and the distribution of detail coefficients will have a relatively small standard deviation. On the other hand, when the amount of pilling increases, also the standard deviation will increase as the pills introduce variations into the image that disrupt the underlying pattern of the fabric structure. Referring, for instance, to Figure 17, taken from [33], it can be noticed that for fabrics with pilling grade equal to 1 (in the paper indicated with the letter i) a lower value for standard deviation (i.e. the coefficient $\mathrm{SDc}_{\mathrm{n}}^{\mathrm{h}}$ in the paper) can be found. As the pilling grade rise from 1 to 5 (in the paper from i to $\mathrm{v}$ ), also the $S D c D_{\mathrm{n}}^{\mathrm{h}}$ tends to rise.

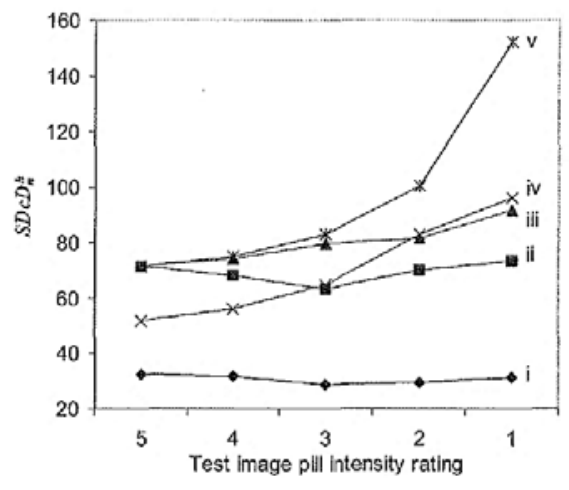

FIGURE 17. Test image pill intensity rating vs. standard deviation $\mathrm{SDc}_{\mathrm{n}}^{\mathrm{h}}$ for five pilling grades from i to $\mathrm{v}$ [33].

However, as stated by the authors, a drawback of this method is that frequency domain analysis cannot provide location information. Moreover, under particular conditions, pilling may be expected to occur periodically, so that it cannot be easily discriminated.

A new approach for pilling evaluation based on the multi-scale two-dimensional dual tree complex wavelet transform (CWT) has been proposed in [34]. The CWT method [35] is used to decompose the pilled fabric image with six orientations at different scales and reconstruct fabric background texture and pilling sub-images. An energy analysis method is, at that time, used to search for an optimum image decomposition scale and to dynamically discriminate pilling image from noise, fabric texture, fabric surface unevenness and brightness variation in the pilled fabric image.

In Figure 18 a 3D mesh plot of WoolMark ${ }^{\circledR}$ SM50 Grade 1 woven fabric is shown, taken by [34]. Using the proposed method it is possible to identify pilling information over a fused and smoothed background of gray value zero at different scales. The positive and negative maximum gray values of the reconstructed detail image represent the highest point of pilling and the deepest point of the pilling shadow respectively. 


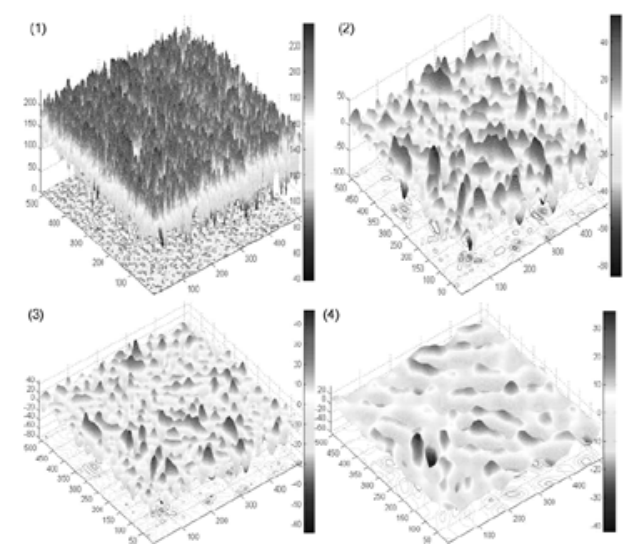

FIGURE 18. 1) 3D mesh plot of WoolMark ${ }^{\circledR}$ SM50 Grade 1 woven fabric from [34]; 2) identified pilling; 3) identified pilling at scale $5 ; 4)$ identified pilling at scale 6.

This approach can be considered hybrid with the ones described in Section 1.4. since a LevenbergMarquardt back-propagation neural rule is finally used to classify the pilling grade. The robustness of the above proposed method has been assessed by Zhang et al. in 2012 [36]. In detail robustness in terms of image rotation, image dilation, image brightness variation and image contrast variations has been assessed. The results provided by the authors suggest that the pilling identification method is robust to significant variation in the brightness and contrast of the image, rotation of the image and dilation of the image. The pilling feature vector developed to characterize the pilling intensity is robust to the brightness change (but sensitive to large rotations of the image). Obviously, it requires all images be arranged such that the illumination is coming from the same direction. As long as all images are adjusted to have the same contrast level, the method provides an objective measurement of the pilling volume and so it can be used to classify the pilling intensity.

\section{D Imaging Methods}

To comprehensively understand fabric pilling phenomena thus allowing an accurate pilling grading, 3-D non-contact scanning systems can be considered better equipment with respect to 2D imaging devices. Actually, these devices are able to evaluate the overall fabric surface unevenness as well as pill characteristics (e.g. pill number, area, and population density). On the basis of this awareness, some recent works have been carried out in order to assess pilling grading of fabrics (see Figure 19) using 3D vision. In fact, at the beginning of the 21th century, Sirikasemlert and Tao [37] described an objective evaluation system for characterizing textural changes in knitted fabrics during simulated wear using 3D devices and, in particular, laser-triangulation technique. The investigated textural changes are pilling, fuzzing, and changes in the constituent yams. Two-dimensional Fourier analysis and wavelet analysis are, then, introduced as new analytical tools for objectively evaluating surface textural changes based on 3D features.

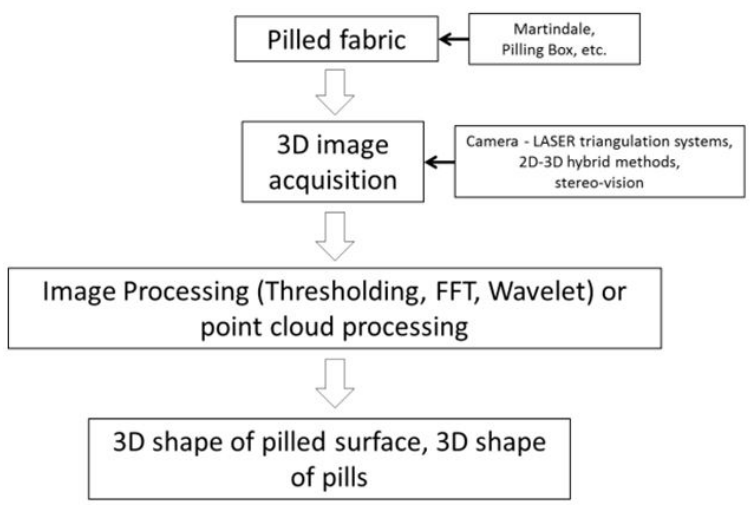

FIGURE 19. Flowchart of 3D imaging methods.

Another important attempt in using 3D imaging for pilling assessment is provided in [38], where a CCD camera was again used to capture the image of a laser line projected on the surface of a series of fabric specimens. Then, making use of trigonometric calculations, the three-dimensional shapes of the inspected fabrics are recovered. Such a 3D reconstruction is eventually used for determining the number, the area, and the density of pills.

The study proposed by Kim and Park [39] is, perhaps, the first attempt in quantifying and evaluating fabric pilling using alternatively twodimensional and three-dimensional imaging methods depending on the kind of base fabric to be examined. Two-dimensional imaging equipment consisting of a CCD camera (640x480 pixels) is used for capturing a square area of $50 \mathrm{~mm}$ for some samples. 2D acquisition is then integrated with a threedimensional measurement obtained by means of the equipment shown in Figure 20.

Various image processing techniques such as FFTbased ones, as well as three-dimensional data processing algorithms, were developed for extracting both pills information and a series of shape parameters, ultimately used for the objective evaluation of fabric pilling. Authors finally state that fabrics with thick and hairy yarn are better assessed using three-dimensional measurement because the sizes of pills are rather large. 

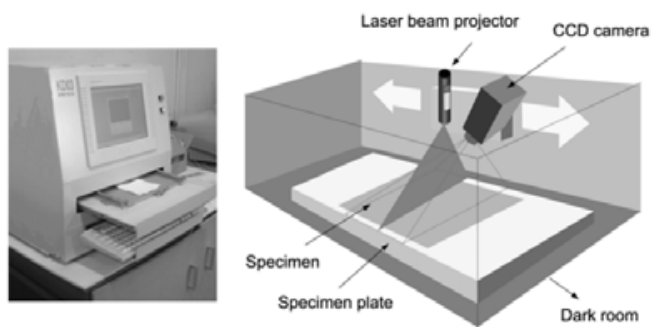

FIGURE 20. Schematic diagram of the 3D measurement system proposed by [39].

On the other hand, fabric with thin and smooth yarn seem unsuitable for this method because the size of pills sometimes went below the intrinsic measurement error of the 3D system and therefore it is necessary to use only 2D-based techniques.

Mettes et al. [40] devised a method based on optical triangulation that performs topographic reconstruction of textile fabric samples. The approach allows the evaluation and the quantification of the pilling formation on the basis of topographic changes. Several tests have been carried out by authors providing robust and precise results.

$\mathrm{Xu}$ et al. [41] introduced a 3D imaging system designed for objective evaluation of fabric pilling. The system was aimed at reconstructing 3D surfaces of fabrics by using two side-by-side images captured by a pair of digital cameras, without special lighting. Once the depth data are captured, the most relevant information for pilling segmentation is identified, and pilling is subsequently measured.

A non-contact method based on laser triangulation sensor capable of measuring the height of the fabrics with five micron accuracy has been developed by Saharkhiz and Abdorazaghi in 2012 [42].

For each sample, 62500 data points were collected and in order to remove noise involved, data a median algorithm for smoothness is applied (see Figure 21).

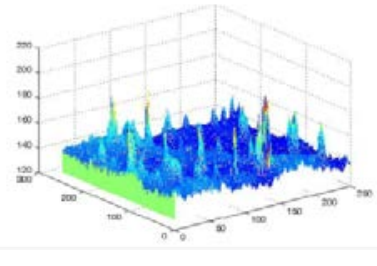

Raw acquired data

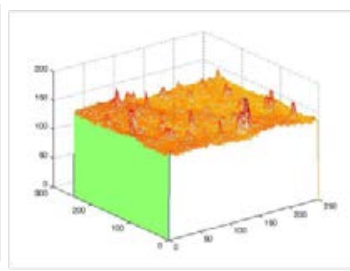

Data processed using median filtering
FIGURE 21. Example of raw data acquired from a fabric using the device described in [42] vs. the same data filtered using a median filter.
Once acquired, a set of pilled fabrics was processed using Median-cut, K-mean and Competitive Learning algorithms in order to extract the number of pills, protruding geometrical volume and area of pills. The comparison among the three above mentioned methods revealed that the correlation factor in objective and subjective evaluation of the pilling of the samples by using Competitive Learning of $\mathrm{K}$ mean algorithm is 0.985 and is more reliable when compared to K-mean and Median-cut methods (see Figure 22).

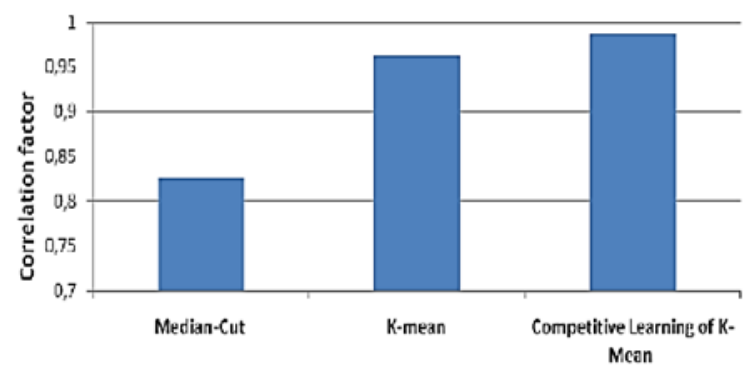

Clustering Method

FIGURE 22. Comparison among correlation factor of objective and subjective fabric pill grading by using Median-cut, K-mean, Competitive Learning of K-mean methods (from [42]).

3D reconstruction of fabric surfaces (including the pills) has also been recently realized in [43] using gradient fields method and starting from a set of four images. Afterward, the pills are detected in 3D fabric surfaces using image-processing tools, typically implemented in the MATLAB ${ }^{\circledR}$ software environment.

Recently, [44] a stereovision system and the threedimensional (3-D) image analysis algorithms for fabric pilling measurement has also been proposed (see Figure 23). Based on the depth information available in the 3-D image, the pilling detection process starts from the seed searching at local depth maxima to the region growing around the selected seeds using both depth and distance criteria.
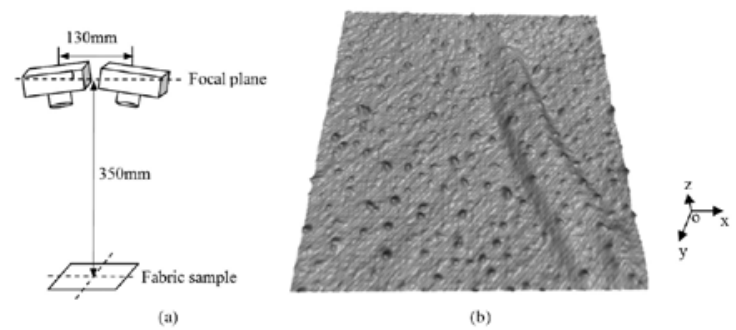

FIGURE 23. a) Illustration of the stereovision system proposed in [44]; b) generated 3D fabric image. 
After the pilling detection, the density, height, and area of individual pills in the image can be extracted to describe the pilling appearance, as shown in Figure 24.

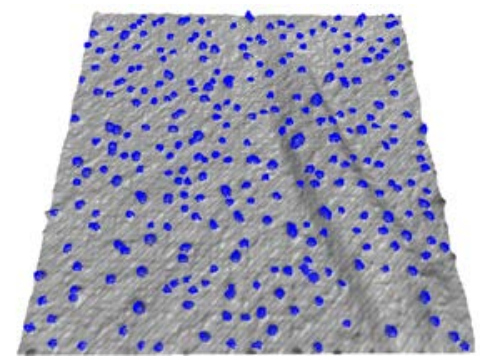

FIGURE 24. Map of detected pilling areas from [44].

\section{Artificial Neural Network-Based Approaches}

Artificial Neural Networks (ANNs) has been extensively used at a research stage in several fields of textile industry since last decades [45-61]. ANNs are particularly applied in spinning, weaving, dyeing and quality control engineering. The massive use of this framework encouraged also the development of methods for pilling evaluation. In detail, ANNs are combined together with image processing in order to strengthen the traditional approaches. The main idea, for this range of works, may be twofold:

1) ANNs may be used as the final phase after the image segmentation (2D or 3D) for correlating the pills typical parameters with the experts' judgment (see Figure 25).

2) ANNs may be used to avoid image segmentation for detecting the pills; fabric images are treated using image processing methods with the aim of extracting significant parameters to be used as a training set for intelligent systems (but not to segment image); consequently, if the starting phase is still image processing, this is used not for separating pills from the background but as a pre-processing step for AIbased methods (see Figure 26).

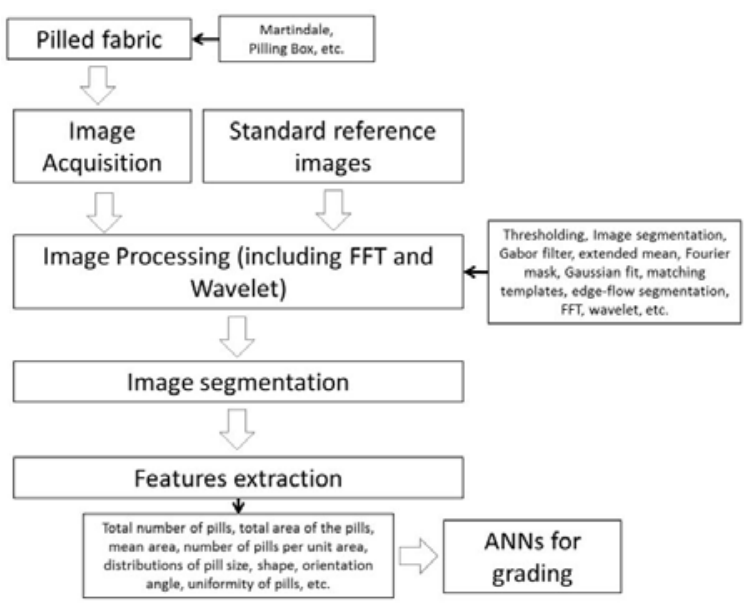

FIGURE 25. Flowchart of ANNs-based methods where ANNs are used in the final stage of pilling grading.

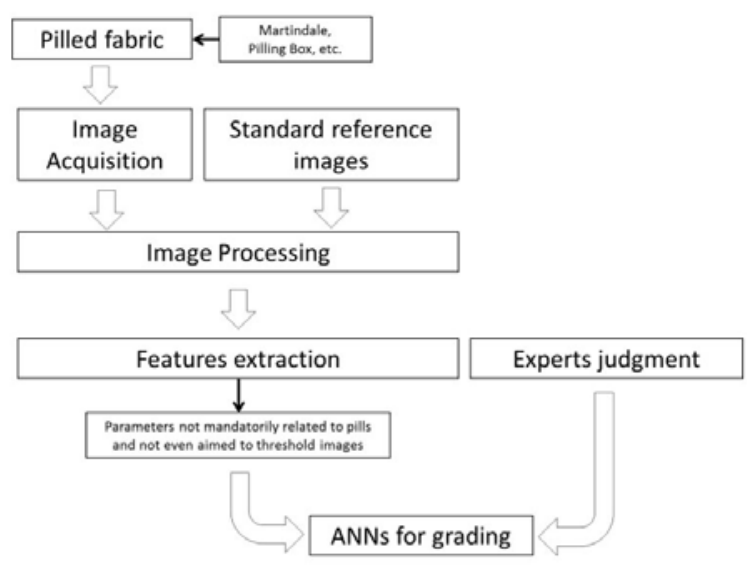

FIGURE 26. Flowchart of ANNs-based methods where ANNs are used for correlating parameters (not necessarily related to pills or coming from image segmentation) with the experts' judgment.

Dealing with the first kind of methods, in [62] Chen and Huang evaluated and graded fabric pilling based on light projection using image analysis and neural network to overcome the common difficulty of interference with fabric pill information from fabric color and pattern. Pilling grade is assessed by using a Kohonen self-organizing feature map [5] neural network. Thirty different kind of pilled fabric samples were trained and tested, and the correlation coefficient between the objective grade and subjective grade is 0.94 for the training samples and one for the testing samples. The sample number with \pm 1 grade deviation is 5 , so the objective inspection accuracy is stated equal to $83 \%$. 
In [63] an energy analysis method to search for an optimum image decomposition scale and dynamically discriminate pilling image from noise, fabric texture, fabric surface unevenness, and illuminative variation in the pilled fabric image is proposed. For pilling objective rating, six parameters were extracted from the processed images to describe pill properties. Finally, a Levenberg-Marquardt back propagation neural rule was used as a classifier to classify the pilling grade. The proposed method was evaluated using knitted, woven, and nonwoven pilled fabric images acquired by a digital camera.

In Xin's PhD dissertation [64] the results obtained in [21] were used as a training set for three kinds of ANNs in order to correlate the extracted parameters with the expert's judgment. Results show a correlation coefficient equal to 0.9358 .

Dealing with to the second kind of methods, in [65] a feed forward back-propagation artificial neural network (FFBP ANN) has been trained to determine the degree of pilling (i.e. to classify the fabric into a number of quality classes). Images of pilled fabric samples are acquired using an appositely devised machine vision system. Then, the acquired images are pre-processed in order to 1) discard color information, 2) to correct non-uniformity in illumination and 3) to extract 11 parameters describing pilled fabrics (see Figure 27).

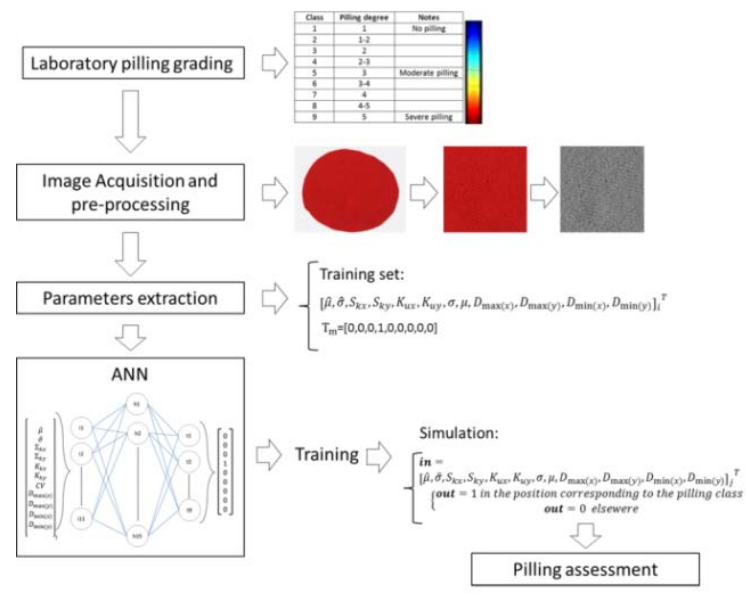

FIGURE 27. Flow diagram of the procedure proposed in [65].
As mentioned above, such parameters are used for training the ANN which is finally used as a tool for assessing the degree of pilling of new fabric samples. The work is inspired by a previous one by the same authors [5] whose aim was to detect and classify a number of defects possibly occurring on raw fabrics such as stains, thin and thick bars, fillings, double fillings, weft threads, double warp threads and broken ends.

\section{CONCLUSION AND DISCUSSION}

From the state of the art analysis, it is evident that 2D imaging systems for pilling measurement are still used nowadays focusing on different, and progressively more precise and reliable, methods aimed at image segmentation. Whichever the image processing-based method for enhancing the pills is, almost all the methods share the idea that the central issue for automatic and objective pilling assessment is to directly measure pills geometric properties. This consideration can be, roughly, confirmed by analyzing the works summarized in Sections 1.1 and 1.2 .

Nonetheless, as described in Sections 1.3 and 1.4, 2D imaging is not the only possible strategy: in the last years a number of works implementing 3D imaging and AI-based systems have been devised in support or in substitution to "traditional" 2D-based methods.

In particular, 3D imaging has been, explored, and still is, for developing pilling measurement methods taking into account the third dimension in the analysis. Since fabric pills have a 3D structure, such methods may be considered a promising frontier for textile control, especially thanks to the decreasing cost of 3D imaging devices. On the other hand, if on one side ANN-based methods are not new in the textile field, their application in pilling measurement (especially regarding pilling grading) is far to be fully explored and relevant works were carried out only in the last 10 years. As a consequence, in authors' opinion, it is highly advisable to investigate more thoroughly methods which make use of these techniques. Moreover, it is necessary to favor more integration between imaging systems and AI-based ones.

In Table II, the most relevant works are synoptically proposed divided into 4 periods: 1990 - 2000, 20012005, 2006-2010 and 2011-2014. 
TABLE II. Most important works dealing with pilling measurement.

\begin{tabular}{|c|c|c|c|c|}
\hline & $1990-2000$ & 2001-2005 & 2006-2010 & 2011-2014 \\
\hline \multirow{7}{*}{$\begin{array}{l}\text { 2D imaging Methods } \\
\text { based on thresholding }\end{array}$} & $\begin{array}{c}\text { Konda et al. } \\
1990\end{array}$ & \begin{tabular}{|c|} 
Jensen et al., \\
2002
\end{tabular} & \multirow{3}{*}{$\begin{array}{l}\text { Naderpour et } \\
\text { al., } 2009\end{array}$} & Gao et al., 2011 \\
\hline & $\begin{array}{c}\text { Abril et al. } \\
1996\end{array}$ & $\begin{array}{l}\text { Xin et al., } \\
2002\end{array}$ & & \multirow{6}{*}{ Jing et al., 2011} \\
\hline & Annis, 1996 & \begin{tabular}{|c|} 
Behera et al., \\
2005
\end{tabular} & & \\
\hline & $\begin{array}{c}\text { Abril et al. } \\
1997\end{array}$ & \multirow{4}{*}{$\begin{array}{c}\text { Huang et al., } \\
2005\end{array}$} & \multirow{4}{*}{$\begin{array}{c}\text { Xiaojun et al., } \\
2009\end{array}$} & \\
\hline & \begin{tabular}{|c|} 
Xiaohong \\
and Mu, 1998
\end{tabular} & & & \\
\hline & His et al. 1998 & & & \\
\hline & \begin{tabular}{|c|} 
Fazekas et al., \\
1999 \\
\end{tabular} & & & \\
\hline \multirow{4}{*}{$\begin{array}{l}\text { 2D imaging Methods } \\
\text { based on Fourier } \\
\text { analysis and wavelet }\end{array}$} & & \multirow{3}{*}{$\begin{array}{l}\text { Xin et al., } \\
2002\end{array}$} & \multirow{2}{*}{$\begin{array}{l}\text { Palmer et al., } \\
2009\end{array}$} & Deng et al., 2011 \\
\hline & & & & \multirow{3}{*}{ Zhang et al., 2012} \\
\hline & & & & \\
\hline & & \begin{tabular}{|c|} 
Behera et al., \\
2005
\end{tabular} & Jasińska, 2009 & \\
\hline \multirow{4}{*}{ 3D imaging Methods } & & \begin{tabular}{|c|}
$\begin{array}{c}\text { Sirikasemlert } \\
\text { and Tao, } \\
2000\end{array}$ \\
\end{tabular} & $\begin{array}{c}\text { Kim and Park, } \\
2006\end{array}$ & Xu et al., 2011 \\
\hline & & $\begin{array}{l}\text { Kang et al., } \\
2004\end{array}$ & \multirow{3}{*}{$\begin{array}{c}\text { Mendes et al., } \\
2010\end{array}$} & $\begin{array}{c}\text { Saharkhiz and } \\
\text { Abdorazaghi, } \\
2012\end{array}$ \\
\hline & & $\begin{array}{c}\text { Kim and } \\
\text { Kang, 2005 }\end{array}$ & & Techniková et al., \\
\hline & & \begin{tabular}{|c|} 
Mettes et al., \\
2006
\end{tabular} & & 2013 \\
\hline \multirow{4}{*}{ AI-Based methods } & & & \multirow{4}{*}{$\begin{array}{l}\text { Chen and } \\
\text { Huang, 2004 }\end{array}$} & $\begin{array}{c}\text { Behera and } \\
\text { Mishra, 2006 } \\
\end{array}$ \\
\hline & & & & Deng et al, 2009 \\
\hline & & & & Xin, 2009 \\
\hline & & & & Furferi et al., 2014 \\
\hline
\end{tabular}

From this time analysis, the following considerations can be drawn:

- after a strong initial impetus in the years 1990-2000, 2D imaging methods have not experienced a shutdown, and presumably more methods will be devised in the near future;

- Fourier and wavelet analysis will probably be further investigated for pilling grading systems, since they are relatively new techniques applied to this field;

- 3D methods will probably replace some of the less promising 2D imaging methods in the near future;

- ANN based methods were developed fairly late, as a consequence future developments are expected in this field.

Finally, few comments can be drafted regarding the costs of equipment for assessing pilling grade on textiles. Systems based on 2D images are required to perform image analyses using a sufficient resolution. Due to small dimensions of pilled areas (e.g. diameter equal to about $1 \mathrm{~mm}$ ), acquiring devices should be chosen in order to acquire a single pill using at least 50 pixels. This means that the minimum required spatial resolution has to be equal to 0.02 mm/pixel. Accordingly a camera with a 2048 x 1536 pixels resolution is recommended for $38 \mathrm{~mm}$ diameter specimens. To date, the cost for a 3.1 MPixel commercial camera is more than affordable.

3D imaging based methods, on the other hand, involve higher costs since commercial 3D scanners are still expensive. Even in case the textile company wishes to create its own 3D acquisition system, the costs increase since at least two cameras or a camera and a Laser beam projector are required.

Regarding the scene illumination, it could be useful to use D65, D50 or TL84 illuminants, thus involving a further cost (often higher than the one associated to the $2 \mathrm{D}$ acquisition device).

In any case, it has to be noticed that the main costs associated to MV-based methods for pilling assessment are related to the necessary knowledge in creating efficient image processing algorithms; often, this cost dramatically exceeds the one required for vision equipment.

Summing up, the present work presented an overview of the most important works in the field of pilling measurement techniques and methods using MV. Without claiming to be exhaustive, this work is a first attempt in providing a survey of possible approaches dealing with this well-known field of textile research. Since one of the main problems preventing a direct comparison of the performance of the (many) existing methodologies is the lack of tests performed on the same set of specimens, future work will deal with direct implementation of the most promising techniques and their application on a broad set of fabric samples.

\section{REFERENCES}

[1] J-Fan, J., Yu, W.W., Hunte, L., "Clothing appearance and fit: science and technology". Cambridge: Woodhead Publishing Series in Textiles, 2004 No. 33. pp. 54-60.

[2] Gunavathi P., Ragunathan K., "Pilling evaluation: A new method". The Indian Textile Journal, 2008, August 2008 issue.

[3] Abouelela, A., Abbas, H. M., Eldeeb, H.,Wahdan, A. A., Nassar, S. M., "Automated vision system for localizing structural defects in textile fabrics", Pattern Recognition Letters, 2005, 26 (10), pp.14351443. 
[4] Carfagni, M., Furferi, R., Governi, L., “A realtime machine-vision system for monitoring the textile raising process", Computers in Industry, 2005, 56 (8-9), pp. 831-842.

[5] Furferi, R., Governi L., "Machine vision tool for real-time detection of defects on textile raw fabrics", Journal of the Textile Institute, 2008, 99 (1), pp. 57-66.

[6] Furferi, R., Governi, L., Volpe, Y., “A novel method for ring spinning performance evaluation based on Computer Aided analysis of yarn geometry”, International Journal of Mechanics, 2012, 6 (4), pp. 212-221.

[7] Konda, A., Xin, L. C., Takadera, M., Okoshi, Y., Toriumi, K., "Evaluation of pilling by computer image analysis", Journal of the textile Machinery Society of Japan, 1990, 36(3), pp. 96-107.

[8] Abril, H.C., Millan, M.S., Navarro, R.B., "Pilling evaluation in fabrics by digital image processing”, Proceedings of SPIE - The International Society for Optical Engineering, 1996, 2786, pp. 19-28.

[9] Abril, H.C., Millan, M.S., Torres, Y.M., Navarro, R.B., "Image segmentation based on a Gaussian model applied to pilling evaluation in fabrics", Proceedings of SPIE The International Society for Optical Engineering, 1997, 3101, pp. 283-291.

[10] Gonzalez, R.C., Woods, R.E., "Digital Image Processing”, Addison-Wesley, Reading (Mass), 1992. ISBN 0-201-50803-6

[11] Portilla, J., Strela, V., Wainwright M.J., Simoncelli, E.P., "Image denoising using scale mixtures of Gaussians in the wavelet domain”, Image Processing, IEEE Transactions on, 2003, 12 (11), 1338-1351.

[12] Annis, P.A., "Pilling Evaluation of Laboratory Abraded, Laundered, and Worn Fabrics Using Image Analysis”, Book of papers International Conference and Exhibition American Association of Textile Chemists and Colorists, 1996, pp. 465-479.

[13] Iqbal M. D., Mahmood, W., Vachtsevanos, G., "Automated pilling detection and fuzzy classification of textile fabrics", Proc. SPIE 3029, Machine Vision Applications in Industrial Inspection V, 1997, 3029. .

[14] Donald, L., "The Radon transform on Euclidean space", Communications on Pure and Applied Mathematics, 1966, 19(1), pp. 49-81.

[15] Pollock, D.S.G., "Trend estimation and detrending via rational square-wave filters”, Journal of Econometrics, 2000, 99(2), pp. 317-334.

Journal of Engineered Fibers and Fabrics

Volume 10, Issue 3 - 2015
[16] Chen M.S., Wang, S.W., "Fuzzy clustering analysis for optimizing fuzzy membership functions”, Fuzzy Sets and Systems, 1999, 103(2), pp. 239-254.

[17] Hsi, C. H., Bresee, R. R., Annis P. A., "Characterizing Fabric Pilling by Using Image-analysis Techniques. Part I: Pill Detection and Description”, Journal of The Textile Institute, 1998, 89 (1), pp. 80-95.

[18] Wang, X., Yao, M., “Assessing the pilling of fabric by image analysis", Journal of Dong Hua University (English Edition), 15 (4), pp. 68-70.

[19] Fazekas, Z., Komuves, J., Renyi, I., Surjan, L., "Towards objective visual assessment of fabric features”, Image Processing and its Applications, 1999, 1, pp. 411-416.

[20] Jensen, K. L., Carstensen, J.M., "Fuzz and pills evaluated on knitted textiles by image analysis”, Textile Research Journal, 2002, 72 (1), pp. 34-38.

[21] Huang, L., Hong, G., Luo R., "Fabric pilling assessment by digital image processing”, 2005 Beijing International Conference on Imaging: Technology and Applications for the 21st Century, 2005, pp. 168-169.

[22] Xiao, Z.T., Yang, H.W., "Fabric Pilling Segmentation Based On Edgeflow Algorithm”, Proceedings of the Sixth International Conference on Machine Learning and Cybernetics, Hong Kong, 1922 August 2007.

[23] Naderpour, F., Mirjalili, S.A., Sharzehee, M., "The Investigation on the influence of DMDHEU on the Wrinkle and Abrasion Resistance of Cotton Fabrics using Image Processing”, Textile Research Journal, 2009 79, pp. 1571-1577.

[24] Xiaojun, L., Huabing, H., Yushu, L., Hong, Z., "The evaluation system of fabric pilling based on image processing technique", International Conference on Image Analysis and Signal Processing, 2009, pp. 44-47.

[25] Ma, W.Y., Manjunath, B.S., "Edge flow: a framework of boundary detection and image segmentation”, IEEE Computer Society Conference on Computer Vision and Pattern Recognition, 1997, pp. 744-749.

[26] Bianconi, F., Fernández, A., "Evaluation of the effects of Gabor filter parameters on texture classification”, Pattern Recognition, 2007, 40 (12), pp. 3325-3335.

[27] Gao, W., Wang, S., Pan, R., Liu, J., “Automatic location of pills in woven fabric based on Gabor filter”, Key Engineering Materials, 2011, 464, pp. 745-748. 
[28] Izabela J., “Assessment of a Fabric Surface after the Pilling Process Based on Image Analysis”, FIBRES \& TEXTILES in Eastern Europe, 2009, 17(2) pp. 55-58.

[29] Jing, J., Kang, X., "Fabric pilling image segmentation based on mean shift", Communications in Computer and Information Science, 2011, 143 CCIS (PART 1), pp. 80-84.

[30] Xin, B., Hu, J., Yan, H., “Objective evaluation of fabric pilling using image analysis techniques”, Textile Research Journal, 2002, 72(12), pp. 1057-1064.

[31] Behera, B.K., Madan Mohan, T.E., “Objective measurement of pilling by image processing technique”, International Journal of Clothing Science and Technology, 2005, 17 (5), pp. 279-291.

[32] Kim, S.C., Kang, T.J., "Image Analysis of Standard Pilling Photographs Using Wavelet Reconstruction”, Textile Research Journal, 2005, 75, pp. 801-811.

[33] Palmer, S., Wang, X., "Classification of fabric pilling by image analysis”, EMAC 2003 proceedings: proceedings of the Sixth Engineering Mathematics and Applications Conference: University of Technology, Sydney, Australia, 9-11 July 2003, pp. 175180, Engineering Mathematics Group.

[34] Deng, Z., Wang, L., Wang, X., “An integrated method of feature extraction and objective evaluation of fabric pilling”, Journal of the Textile Institute, 2011, 102 (1), pp. 1-13.

[35] Selesnick, I.W., Baraniuk, R.G., Kingsbury, N.C., "The dual-tree complex wavelet transform”, Signal Processing Magazine, IEEE, (2005), 22(6), pp. 123-151.

[36] Zhang, J., Wang, X., Palmer, S., “The robustness of objective fabric pilling evaluation method", Fibers and Polymers, 2012, 10 (1), pp. 108-115.

[37] Sirikasemlert, A., Tao, X., “Objective evaluation of textural changes in knitted fabrics by laser triangulation”, Textile Research Journal, 2000, 70(12), pp. 10761087.

[38] Kang, T.J., Cho, D-H., Kim, S.M., “Objective Evaluation of Fabric Pilling Using Stereovision”, Textile Research Journal, 2004, 74(11), pp.1013-1017.

[39] Kim, S., Park, C.K., "Evaluation of fabric pilling using hybrid imaging methods”, Fibers and Polymers, 2006, 7 (1), pp. 57-61.
[40] Mettes, A.O., Fiadeiro, P.T., Miguel R.A.L., "Three-dimensional surface reconstruction for evaluation of the abrasion effects on textile fabrics", Proceedings of SPIE - The International Society for Optical Engineering, 2006, 6056, art. no. 60560F.

[41] Xu, B., Yu, W., Wang, R., "Stereovision for three-dimensional measurements of fabric pilling”, Textile Research Journal, 2011, 81(20), pp. 2168-2179.

[42] Saharkhiz S., Abdorazaghi, M., "The Performance of Different Clustering Methods in the Objective Assessment of Fabric Pilling”, Journal of Engineered Fibers and Fabrics , 2012, 7(4), pp. 35-41.

[43] Techniková, L., Tunák, M., Janáček, J., "Determination and Comparison of Fabric Pills Distribution Using Image Processing and Spatial Data Analysis Tools", World Academy of Science, Engineering and Technology International Journal of Information Science and Engineering, 2013, 7(10), pp. 4-9.

[44] Ouyang, W., Wang, R., Xu, B. "Fabric pilling measurement using three-dimensional image”, Journal of Electronic Imaging, 2013, 22(4), art. 043031.

[45] Di Angelo, L., Di Stefano, P., “An evolutionary geometric primitive for automatic design synthesis of functional shapes: The case of airfoils", Advances in Engineering Software, 2014, 67, pp. 164-172.

[46] Furferi, R., Governi, L., Volpe, Y., "Modelling and simulation of an innovative fabric coating process using artificial neural networks”, Textile Research Journal, 2012, 82 12), pp. 1282-1294.

[47] Dong, M., Jiang, H.Y., "Fabric defects image filtering method based on rough set and PC neural networks", Advanced Materials Research, 2010, 121-122, pp. 1012-1017.

[48] Oonsivilai, A., Meeboon, N., "Silk texture defect recognition system using computer vision and artificial neural networks", Proceedings of the 2009 2nd International Congress on Image and Signal Processing, CISP'09, 2009, art. no. 5303972.

[49] Furferi, R., Governi, L., Volpe, Y., "Neural network based classification of car seat fabrics”, International Journal of Mathematical Models and Methods in Applied Sciences, 2011, 5 (3), pp. 696-703. 
[50] Malik, P., Sarkar, D., Bagchi, B., Saha, S.S., Majumdar, A., "Application of artificial neural network for the modelling of yarn properties - A review”, Man-Made Textiles in India, 2003, 46 (2), pp. 59-64.

[51] Shamey, R., Hussain, T., "Artificial intelligence in the colour and textile industry", Review of Progress in Coloration and Related Topics, 2003, 33, pp. 33-45.

[52] Kumar, A., "Neural network based detection of local textile defects”, Pattern Recognition, 36 (7), pp. 1645-1659.

[53] Stojanovic, R., Mitropulos, P., Koulamas, C., Karayiannis, Y., Koubias, S., Papadopoulos, G., "Real-time vision-based system for textile fabric inspection”, Real-Time Imaging, 2001, 7 (6), pp. 507-518.

[54] Fan, K.C., Wang, Y.K., Chang, B.L., Wang, T.P., Jou, C.H., Kao, I.F., "Fabric classification based on recognition using a neural network and dimensionality reduction”, Textile Research Journal, 1998, 68(3), pp. 179-185.

[55] Liu, H., Liu, B., Feng, C., "Predictive modeling and empirical analyses of regional silk fabrics price index based on BP neural network”, Advanced Materials Research, 331, pp. 685688.

[56] Majumdar, A., "Modelling of thermal conductivity of knitted fabrics made of cotton-bamboo yarns using artificial neural network", Journal of the Textile Institute, 2011, 102 (9), pp. 752-762.

[57] Mozafary, V., Payvandy, P., Bidoki, S.M., Bagherzadeh, R., "Predicting the influence of seam design on formability and strength of nonwoven structures using artificial neural network”, Fibers and Polymers, 2013, 14 (9), pp. 1535-1540.

[58] Rosa, J.M., Prado, K.R.M., Alves, W.A.L., Pereira, F.H., Santana, J.C.C., Tambourgi, E.B. (2013), “Applying of a neural network in effluent treatment simulation as an environmental solution for textile industry”, Chemical Engineering Transactions, 32, pp. 73-78.

[59] Bhambure, S.D., Dhavale, A.J., Kadole, P.V., Kodavade, D.V., “Artificial neural network \& its applications in textiles", Journal of the Textile Association, 2013, 74 (1), pp. 31-37.

[60] Falomi, S., Malvezzi, M., Meli, E., Rindi, A., "Determination of wheel - rail contact points: comparison between classical and neural network based procedures”, Meccanica, 2009, 44, pp. 661-686.
[61] Allotta, B., Meli, E., Ridolfi, A., Rindi, A., "Development of an innovative wheel-rail contact model for the analysis of degraded adhesion in railway systems", Tribology International, 2014, 69, pp. 128 - 140, 2013.

[62] Chen, X., Huang, X.B., "Evaluating Fabric Pilling with Light-Projected Image Analysis”, Textile Research Journal, 2005, 74(11), pp. 977-981.

[63] Deng, Z., Wang, L., Wang, X., “An integrated method of feature extraction and objective evaluation of fabric pilling”, Journal of The Textile Institute, 2005, 102 (1),.

[64] Xin B., "Characterization of fabric appearance based on image analysis”, Institute of Textiles \& Clothing, The Hong Kong Polytechnic University, Hong Long, 2009.

[65] Furferi, R., Carfagni, M., Governi, L., Volpe, Y., Bogani, P. "Towards Automated and Objective Assessment of Fabric Pilling” (2014) International Journal of Advanced Robotic Systems, 11, Art. No. a171.

[66] ISO-12945-2:2000 Martindale Method with $415 \mathrm{~g}$ loading mass.

\author{
AUTHORS' ADDRESSES \\ Rocco Furferi, PhD \\ Lapo Governi \\ Yary Volpe \\ University of Florence (Italy) \\ Via di Santa Marta 3 \\ Department of Industrial Engineering \\ Firenze ITALY
}

